\title{
Impact of Computer Remote Education on CLL Learning and Solutions
}

\author{
https://doi.org/10.3991/ijet.v13i03.8387 \\ Xuezhou Liu \\ Weifang University of Science and Technology, Weifang, China \\ 1057066375 @qq. com
}

\begin{abstract}
This paper aims at some impacts of computer remote education on the Chinese Language and Literature (CLL) learning of Chinese overseas students with a learning software enabled with remote education as a study case, to conduct a survey and analysis on how this type of learning software serves the students when they study and what is the best commercial learning software in the market by questionnaire survey, statistical analysis and summary methods. On this basis, it is concluded that the CLL learning software plays an important role in improving the learning efficiency and level of Chinese overseas students. But beyond that, the existing learning software in the market still have many limitations. In the end, to make such learning software play a better role in overseas students' learning process, this paper proposes strategies for upgrading software in question by virtue of operating and popularizing more mature English learning software with remote education function, which provides a valuable reference for future improvement and promotion of similar software
\end{abstract}

Keywords-Computer Remote Education, Chinese Language and Literature, Investigation and Analysis, Solution Analysis

\section{Introduction}

The development of digital and network technologies has ushered people in a new era of computer. In relation to the traditional television and newspapers, computers feature the pluralized content of communication, high interactivity and selfdetermined participation, etc. People can easily and quickly access to computer-based resources and information [1] It is an irresistible trend that computer technology has been widely applied in various fields.

The emergence of computer technology will also make it possible to enable the remote education. Today, a variety of learning software with remote education function have gradually sprung up, especially some mobile devices such as tablet PCs and cellphones which emerge in large numbers such that the learning software is no longer limited to PCs side. In recent years, with the increasing demands for CLL learning and the popularization of computer and network technologies, many learners resort to this type of learning software with remote education and regard them as an important 
tool for improving their CLL learning levels. The demand of learners for Chinese language and literature learning software is on the rise, but such learning software is still in its infancy [2]. Only a limited number of them can be found in the market but immature with a lot of deficiencies and limitations.

In order to explore what is the impact of the computer remote education software on the Chinese language learning of Chinese overseas students and how to improve its effect better, this paper conducts a survey on three fronts such as the objective conditions, situations under which Chinese overseas students use the CLL learning software and their evaluation on it. Then it is found that this learning software has a high tutorial function during students' learning. The sample survey reveals the limitations of all types of existing CLL learning software. In the end, a similar remote education learning software is used for reference to put forward appropriate upgrading strategy [3], which provides a reference base for the development of CLL remote education software.

\section{Survey and Analysis of impact of computer remote education on Chinese language and literature learning}

\subsection{Service conditions the Chinese overseas students use CLL learning software}

Data sources. In order to better understand the impact of learning software on the Chinese language and literature learning, this paper make a survey on those Chinese overseas students at Beijing International Education Institute about objective conditions, situations and evaluation of software services. In this process, 100 questionnaires were distributed to the students in three levels of primary, middle and advanced classes at a rate of 4: $3: 3$ [4]. At last, the 93 valid questionnaires in total were collected.

Analysis of survey results.

What are the number of downloaded CLL learning software and their in-service time. The survey results show that the students are all well qualified to meet the objective conditions of using learning software, that is, they have mobile devices such as a computer or a tablet PC, which has laid a better foundation for the CLL learning software. The number of downloaded CLL learning software in the survey is shown in Fig. 1. We can see from the figure that $74 \%$ of the respondents have installed 1-3 types of software and $26 \%$ of respondents have downloaded 1-8 types or more.

As shown in Fig. 2, 72\% of students use the software for less than 2 hours, while most students access the software by their own search on the network, only some by other referrals. They are applied mostly in dormitory or in study room [5], which implies that the majority of the students are willing to actively use CLL learning software to study Chinese. 


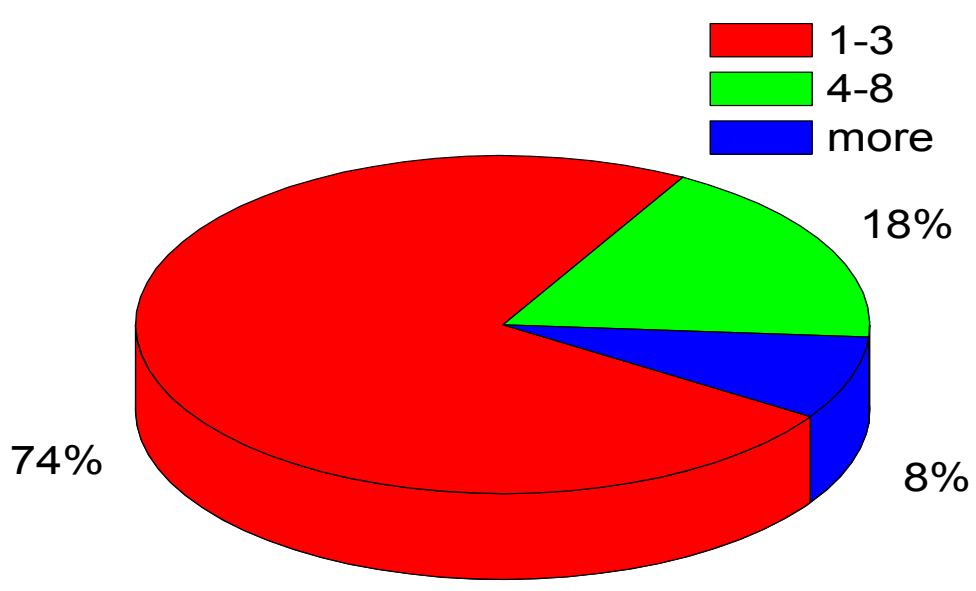

Fig. 1. Download APP quantity distribution

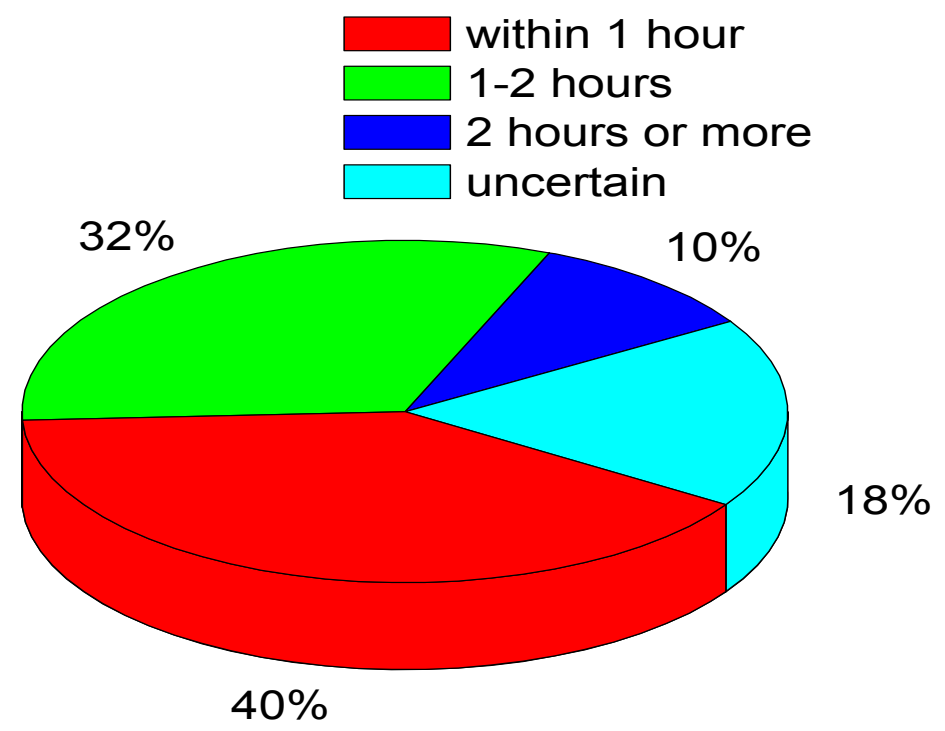

Fig. 2. Use APP duration distribution

What are the content of CLL learning software. As for the main content of CLL software, most of the students use it to look up words and take an examination, the results are shown in Fig. 3. What the students learn from such software also depends on their different levels. More contents are designed for primary learners than for the other two types of learners, while senior learners primarily use tool-based CLL learning software [6]. It follows that the different requirements of learners at all levels for improving Chinese language and literature lead to the diverse content of Chinese language and literature learning software. 
Do students concern about whether the contents of CLL learning software and classroom instruction are synchronized. The survey results in Fig. 4 show that those who choose to be more and very concerned about it account for higher proportion, especially those beginner learners, who show more dependent on classroom instruction, while those who do not concern about and completely, most are mostly middle and senior learners, which implies that they have a certain foundation of Chinese language and literature, and expect to seek more abundant learning resources and content.

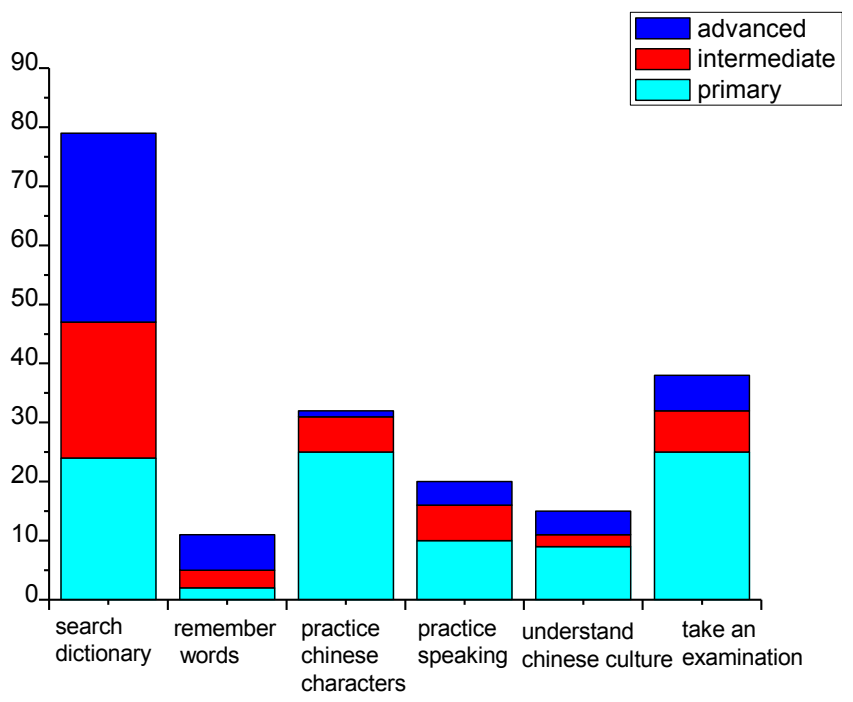

Fig. 3. App software learning content

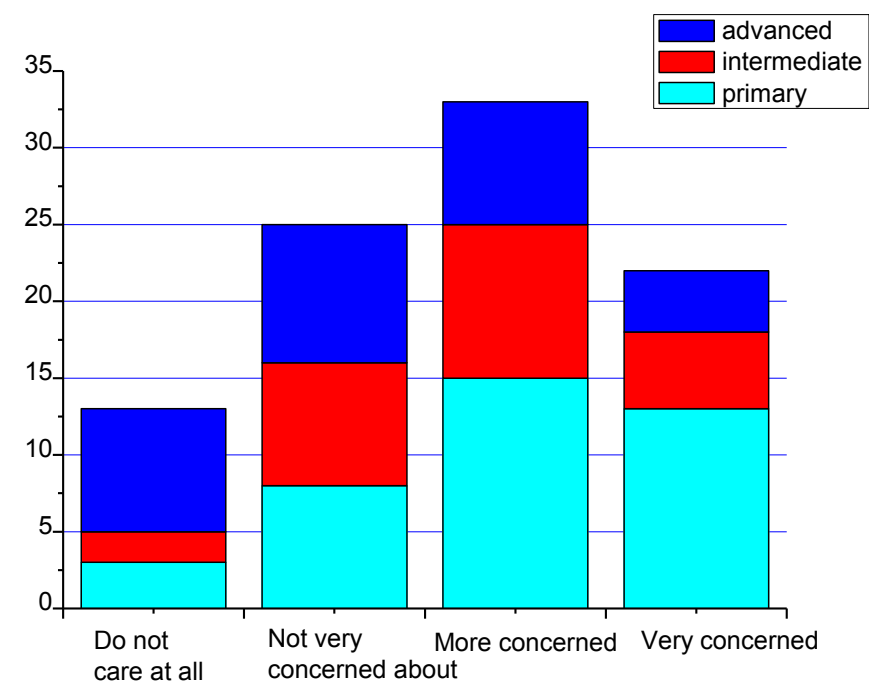

Fig. 4. Do you care about APP content and classroom teaching content is synchronized 
What is the role of CLL learning software in the study. As can be seen from the survey results in Fig. 5, most of Chinese overseas students think that this type of software plays a great or a certain effect on Chinese language and literature learning, and only a few middle and senior students think it does no effect or no obvious effect. This coincides with previous findings. In general, Chinese overseas students hold a positive attitude towards Chinese language and literature learning software.

Why CLL learning software is discarded. As can be seen from Fig. 6, 52\% of overseas students have discarded this type of software for they think there is no any new or alternative function or no any effect in this software, which implies that they show more concern about its function experience and learning effect, regardless of its shortterm attractiveness.

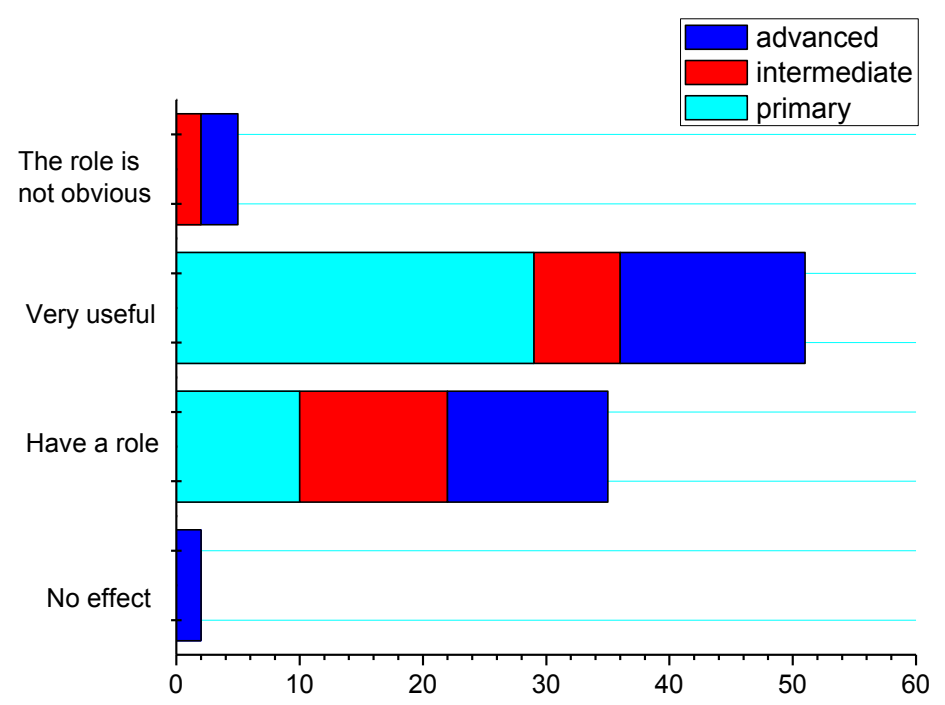

Fig. 5. The Effect of APP Software on Chinese Learning

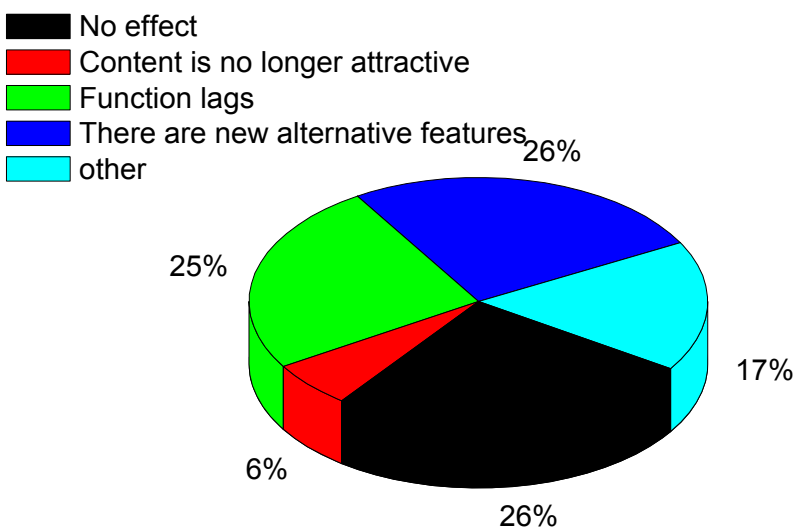

Fig. 6. The main reason APP deprecation 
What features students hope the CLL learning software should have. As can be seen from the survey results in Fig. 7, most of the students show no concern about the types of Chinese language and literature learning software, but more about whether the content is abundant and whether it helps take the exams and tutor them in Chinese language and literature learning. In addition, learners at different levels also have different requirements on its functions. Beginners more focus on the tutorship and taking the examination while middle and senior learners turn to content enrichment and taking the examination [7], which shows that the higher the level of Chinese language and literature, the richer the demand for learning resources.

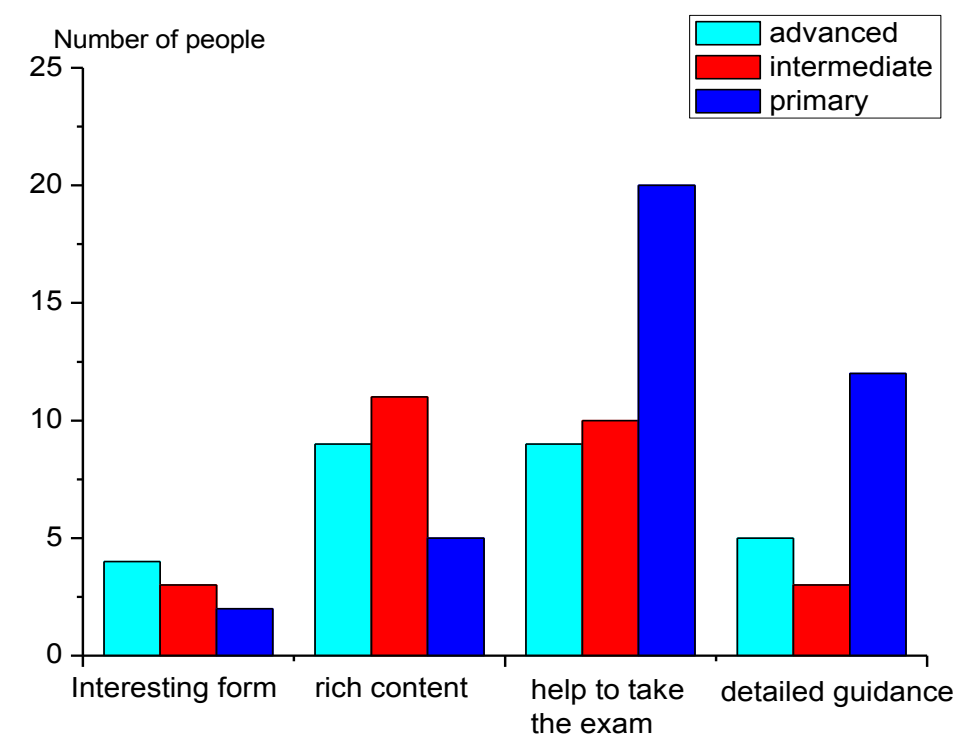

Fig. 7. Expectations for APP

The above findings inform us that the overseas students hold a positive attitude towards the CLL learning software in the study of relevant subject and expect much of the function and content of the CLL learning software.

\section{$3 \quad$ Application and upgrading strategy of CLL learning software}

\subsection{Investigation and analysis}

Data source. With the popularization of mobile devices, CLL learning software with remote education function is no longer limited to the PC side, its APP can be downloaded on the mobile device for application with identical functions. In order to have insight into the current Chinese language and literature learning software, this paper takes 35 similar software available from 932 search results hit by inputting keywords Chinese Language and Literature Learning Software in different kinds of 
languages on major Websites and App Stores, in accordance with Apps Ranking and using the stratified sampling approach [8], as the survey samples. The classification of software is shown in the Table 1.

Table 1. Chinese learning APP classification

\begin{tabular}{|c|c|}
\hline Teaching content & $\begin{array}{l}\text { Tools, Language and Culture, Skill training class (voice, vocabulary, } \\
\text { Chinese, grammar, listening) }\end{array}$ \\
\hline $\begin{array}{l}\text { Production of the main } \\
\text { body }\end{array}$ & Business organizations, training institutions, small studios, individuals \\
\hline Media Voice & Chinese, English, other languages \\
\hline
\end{tabular}

\section{Analysis of survey results.}

Distribution of software providers. In Fig. 8, the survey results show that $72 \%$ of CLL learning software is produced by the commercial institutions, which only involves part of and not focuses on the Chinese language and literature instruction; $14 \%$ are provided by the educational institutions and the individual makers, however, with different core functions. The former provider focuses on examinations, while individual-produced software attaches more importance on semantic or contextual competence training [9].

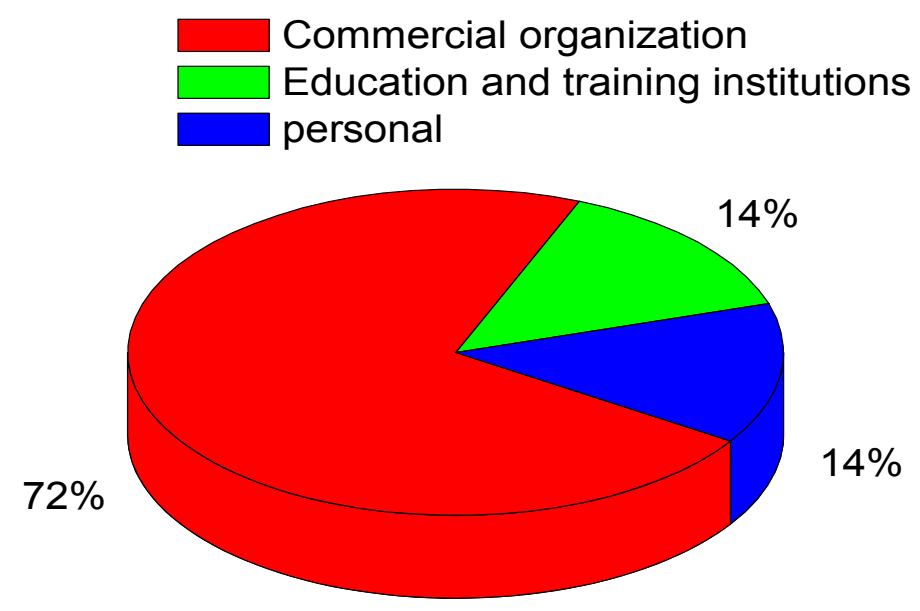

Fig. 8. Production of the main distribution

Media voice and presentation. The survey results, as shown in Fig. 9, tell us that the majority of CLL learning software uses English as a media language, which also has concern with the fact that English is a universal language in the global. In addition, $17 \%$ of them uses multilingual media voices, this more reasonable and humanized design should be the future development trend of Chinese language and literature learning software. 
As can be seen from the survey results in Fig. 10, on the whole, CLL learning software resources are presented in a variety of ways, of which, text, audio and video modes are common used [10], since they can intuitively reflect the meaning, semantics and so on, just as Chinese-language learners require.

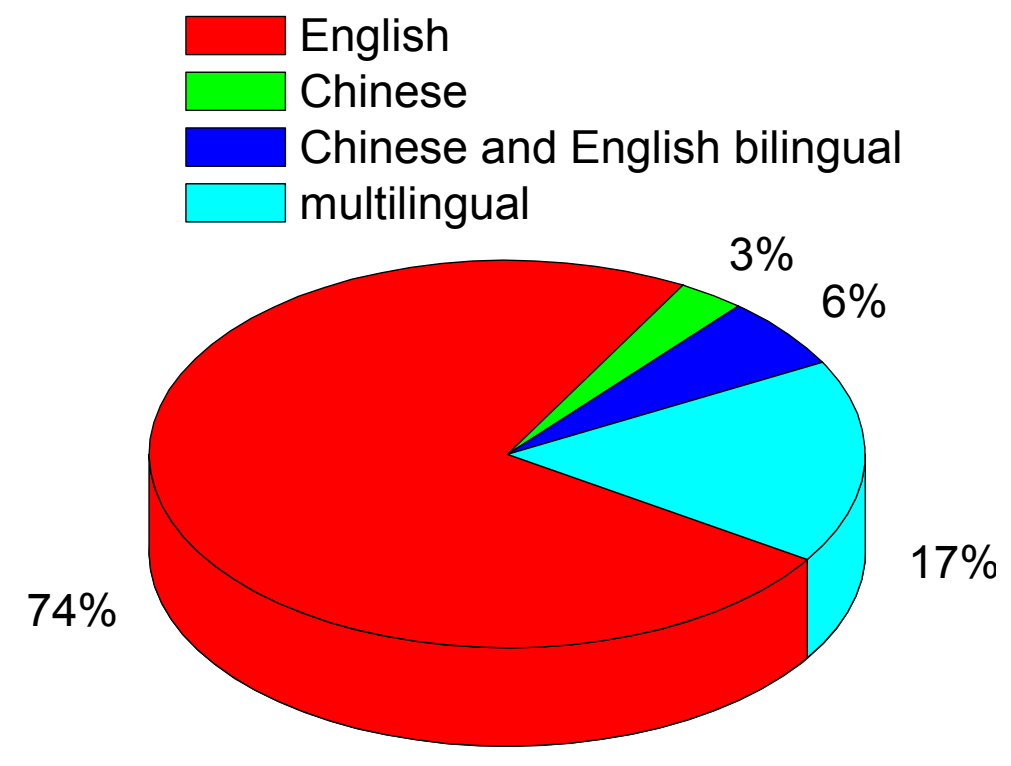

Fig. 9. Media Voice

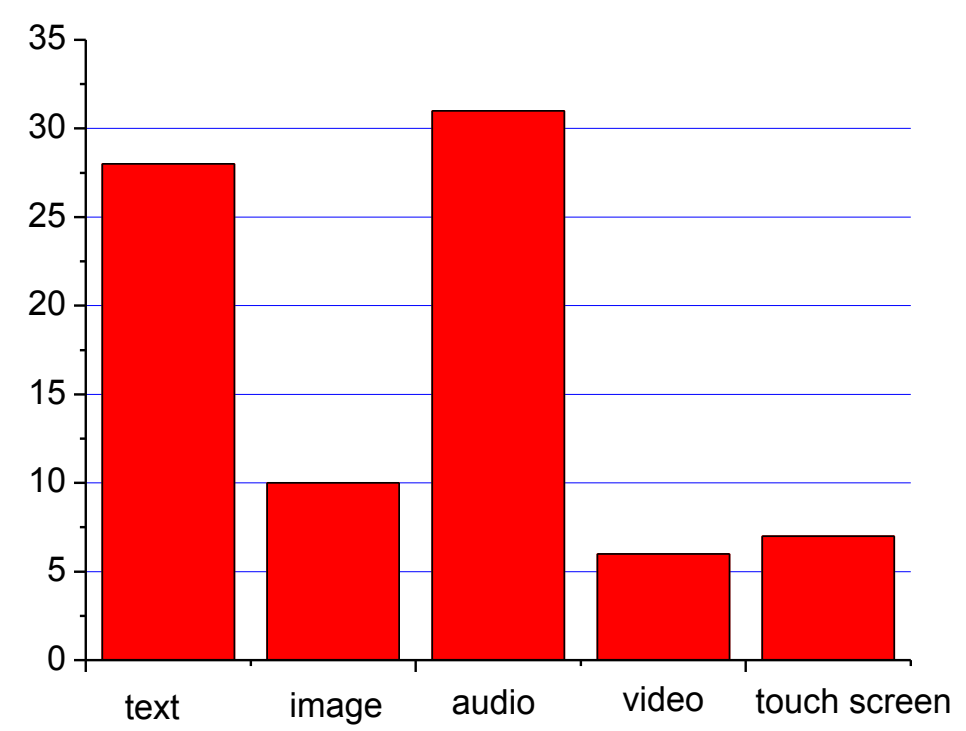

Fig. 10. Rendering mode usage 
Charges, networking, updates and misc. items. As can be seen from the survey results in Fig. 11, 83\% of CLL learning software is free of charge for downloads. As the producer profits from advertisement pushes when users download and use it, current users give bad comments on it and even uninstall it. In the operation process of this kind of software, most of them must be used in a networking environment, therefore off-line application will be enabled as one of the main advantages of CLL learning software [11]. Most software alike is retarded to update, which will also affect the use of user's.

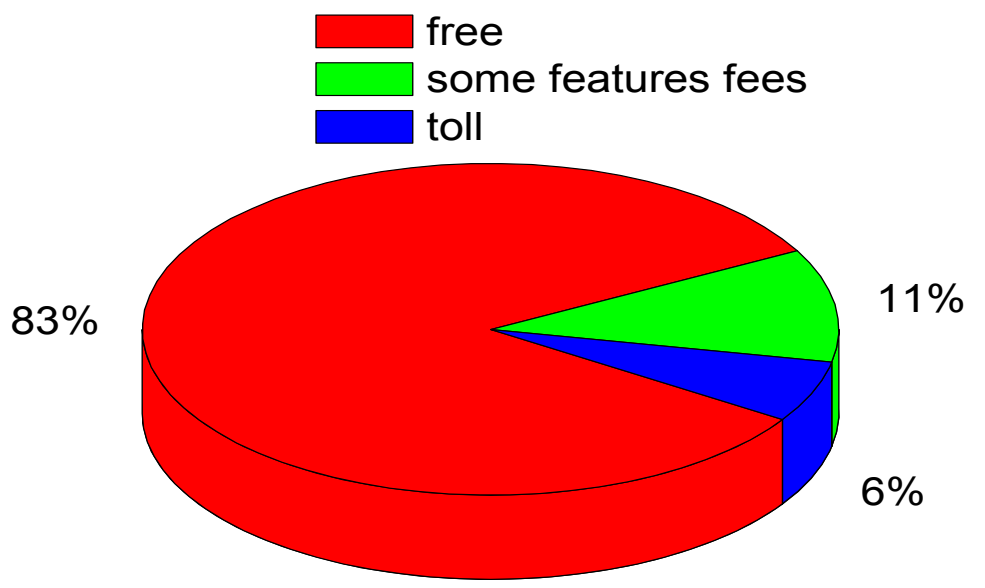

Fig. 11.Sample APP fees

Misc. In this paper, we also make some relevant survey on interface design, interaction and demand orientation aiming at this type of learning software. We discovery from the survey that the different types of CLL learning software presents different effects in the interface design. Some is relatively simple, only include two parts, i.e. the setting and operation interfaces, while some feature more beautiful interface with a strong sense of design and many learning modules for preference, along with a game interface for learning games. This design will help improve the user experience [12]. Currently, most CLL learning software have an obscure positioning match, so that it is difficult to find a matching software by search engine. They also lack of interactivity. Only a few enable a discussion forums and message boards, but still need to be improved in terms of learning plans, progress and other inherited function.

\subsection{Limitations of Chinese Language and Literature Learning Software}

By analyzing the results of survey on how CLL learning software serves the overseas students and what are the current conditions for Chinese language and literature learning software, we found that there are some limitations in the existing Chinese language and literature learning software: 
1. Lack of systematic and targeted contents: Today, most CLL learning software in the market focus on skill training of one aspect (such as word meaning and semantics), and are only applicable for some learners, not meeting the requirements of the learners at all stages.

2. Immature interaction: The interaction mode of Chinese-Chinese language and literature learning software is only limited to simple leaving messages, e-mail and other functions, but the participation degree needs to be improved [13].

3. Limited promotion channels and single mode: Most of the learners adopt CLL learning software after referrals of teachers or their classmates for three reasons as follows, there are relatively less CLL learners; this kind of software is not well known; and Chinese language and literature learning software are immature in operations and promotion [14].

4. CLL learning software varies greatly in quality: The purpose of most CLL learning software produced by commercial institutions is to profit from Ads push after users are attracted to download it. These types of software only focus on formal innovations and lacks the design of instruction contents. Some software produced by special training institutes are highly professional, but due to imitations of studios and individuals, they serve few users and are thus spoiled by a bulk of inferior learning software.

\subsection{Upgrading strategies for English learning software}

In order to improve the quality of Chinese language and literature learning software, this paper, by taking the more mature English learning software with remote education as a study case, using the advantages of its operating mode for reference, proposes some relevant upgrading strategies for it.

Reference significance of English learning software. As an international language, the English has its learning software with remote education function developed more maturely. There are English PC-side and mobile client set up on the majority of learning Websites, rather than those CLL learning software which will be available only by searching. What's more, the recommended modules are classified in detail.

Based on the Apps rankings and ratings of websites, forums, and English learning software, this paper takes the English version of the popular "Gladiator-Study English for Beginner" as an example, bases itself on the common features of English and Chinese languages to analyze it on three fronts so as to provide the reference for improving the CLL learning software. In the view of the popularization and application of mobile devices, this paper takes the remote education enabled on this software on the mobile client as a study case to carry out analysis, which has an identical function with that PC-side learning software.

Demand orientation. In the design, this type of software fully allows for the demands of learners at different stages. The beginners can create their own English learning schedule according to their own circumstances, as shown in Fig. 12. It covers the learning contents of all stages from the elementary school to TOEFL, IELTS.

The learning model in the software is to recite words with the aid of the pictures. As shown in Fig. 13, after entering the "Ready to Study" interface, you can select the 
words to be studied according to the example sentences for learning words. It can also provide word gestures, pronunciation, example sentences and other contents. User may review the previous contents, as shown in Fig. 14, you select in what you are interested for review, such as spelling, gap filling, shorthand with voice, etc., which not only reflect the humanized design, but also enables the learner to improve the efficiency of English learning by repeated training process.

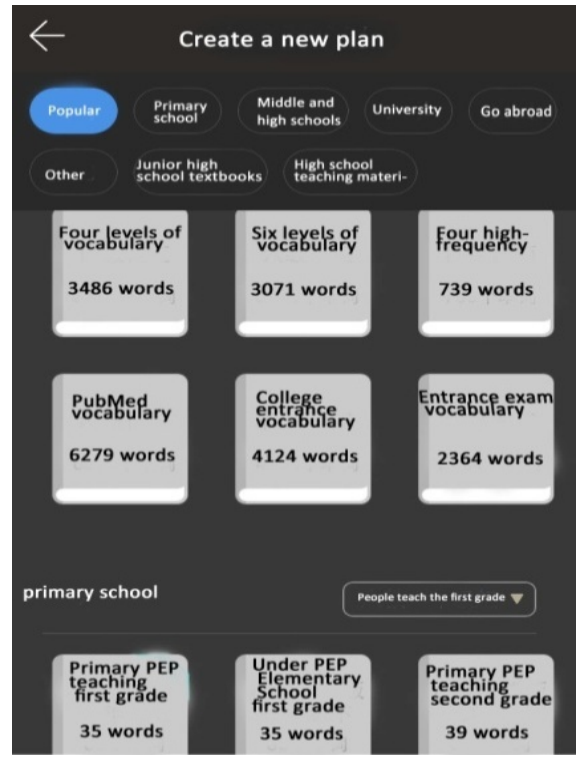

Fig. 12.Create a learning plan

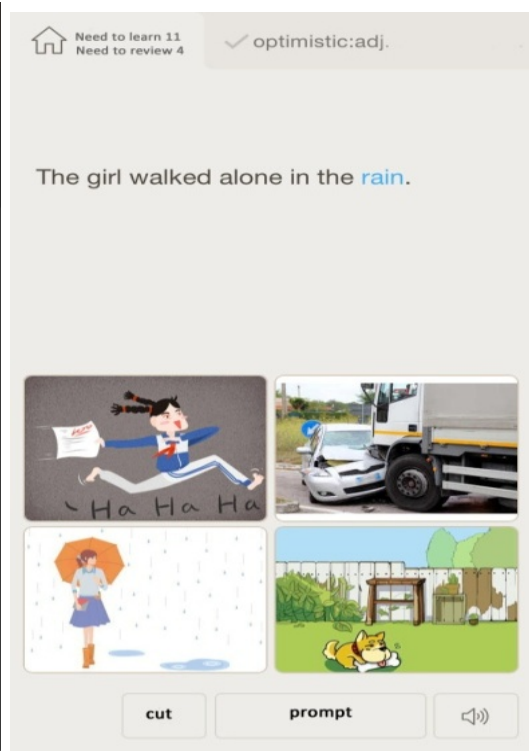

Fig. 13. Learning interface

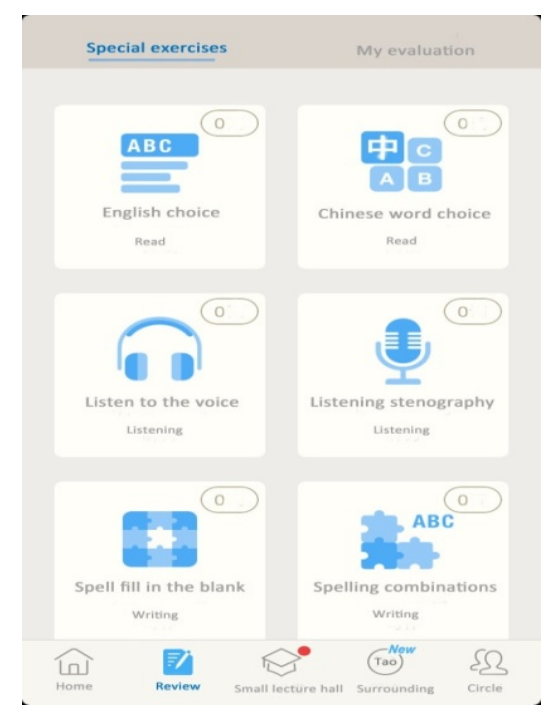

Fig. 14. Review interface 
Interaction mode. A good interaction mode can effectively stimulate and motivate learners' interests. After choosing the Circles function, you can go to a circle that interests you or join the primary class to learn. As shown in Fig. 15, the user can also initiate a PK on word memory with an opponent you have chosen by means of a game. As shown in Fig. 16, the user can also share their learning results by some social network platforms such as WeChat, QQ, etc. in the circle. This design can not only make study fun, but also arouse the learning enthusiasm of learners, while free popularization of learning software is allowed.

Popularization mode. Unlike other charging or AD push modes, this software counts on a self-supporting "Gladiator-Study English for Beginner" brand Flagship store to profit by selling some relevant learning resources, pro forma materials and learning supplies after a steady stream of users is fostered by providing a large amount of free learning resources. As shown in Fig. 17, in doing so, the profitability of software can be guaranteed while meeting the needs of users, more importantly, a greater space can be saved for further popularization of learning software.

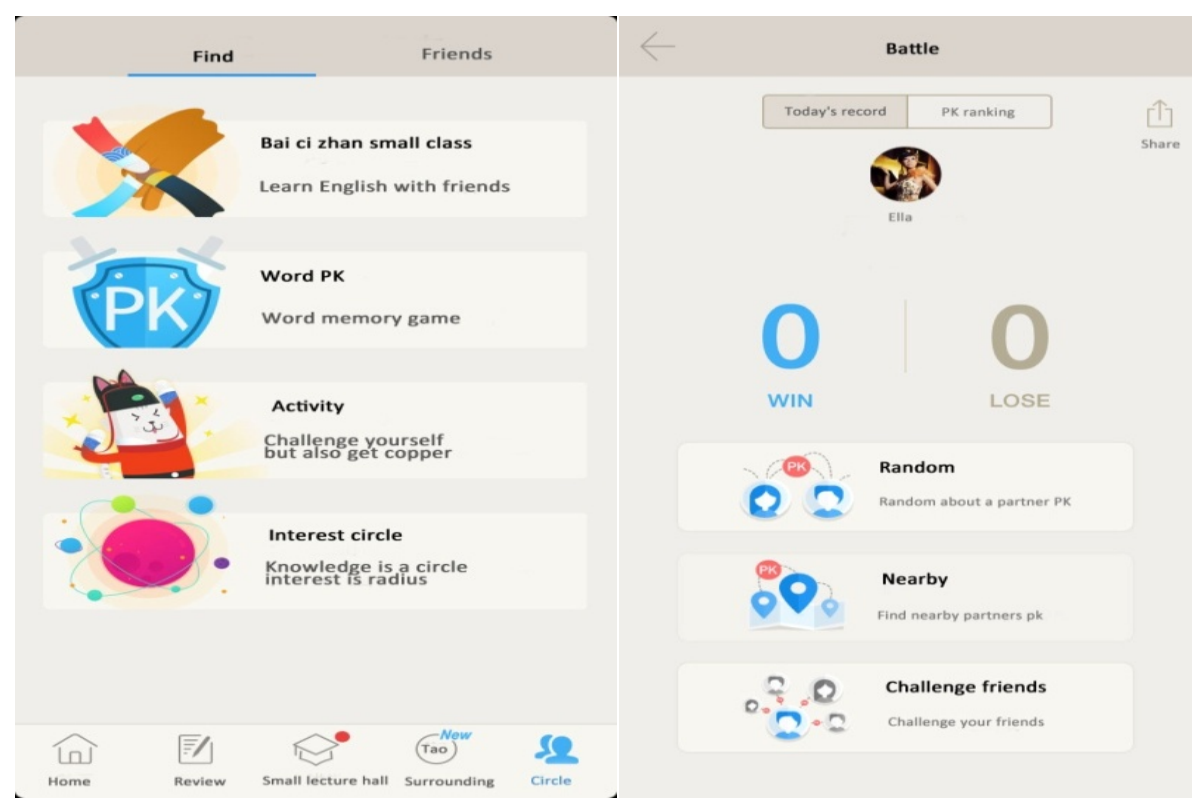

Fig. 15. Circle interface

Fig. 16. Battle interface 


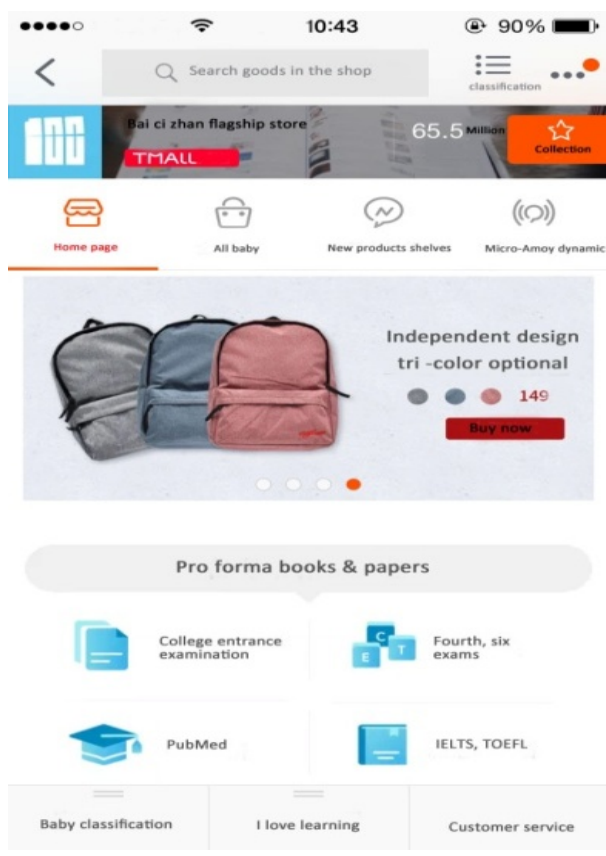

Fig.17. Promotion mode interface

Upgrading strategy for CLL learning software. As there are some limitations in Chinese language and literature learning software, by drawing on the features of more mature English learning software, this paper proposes some strategies for upgrading this type of software as follows:

Accurately target users. Aiming at the systematic process of Chinese language and literature learning and the actual demands of learners for learning this subject, this paper designs a well-positioned Chinese language and literature learning software. For example, most beginners mainly depend on the system of textbooks, focusing on practicing lexical and semantics; while for middle and senior learners who have certain requirements on the history and culture of Chinese language and literature. Others who require some more practical proverbs and literary common senses for work and other reasons [15]. In the design, it should allow for the demands of different stages and population, to avoid users to abandon learning software halfway.

Multi-platform popularization channels. The survey results as above show that the current channels for popularizing the Chinese language and literature learning software are simple, most of them are available through search or referrals. According to the characteristics of CLL learning software, it is suggested in this paper that the promotion channels be broadened from the following aspects: 1) Use Q \& A and search platform to set up software search terms for leading potential users; 3) Use new media resources commonly used by learners like QQ, WeChat, etc., set up the relevant public WeChat, regular post Chinese related articles to this software.

Building diverse interaction platform. Diversified interaction platform, in addition to feedback for users' demands, have the function for operation and promotion this 
type of software. It can be designed to allow user to communicate with designers by using the traditional e-mail, forms. Learners can be allowed to share their own learning progress and results on the microblog, WeChat, QQ and on the other social network platforms while the free popularization is aimed. Certainly, an interaction module in the site should be established to allow students to discuss, also facilitate operators to retrieve relevant information for system updates and improvement. Only in this way can this learning software avoid to be abandoned.

\section{Conclusion}

This paper draws the following conclusions from survey and analysis of the service conditions of CLL learning software with remote education in allusion to the current Chinese language and literature learning software in the market:

1. Based on survey and analysis of the Chinese language and literature learning software with remote education used by the overseas students, it is concluded that this type of software can play a tutorial role and improve the learning efficiency in learning process with an obvious effect.

2. From survey and analysis of Chinese-Chinese language and literature learning software at present, the results show that the development and operation mode of Chinese-Chinese language and literature learning software are not yet mature enough. There are many defects to be improved.

3. In order to promote and apply Chinese language and literature learning software with remote education function, and better serve the overseas students, the "Gladiators-Study English for Beginner" for mobile client is used for reference to propose the upgrading strategies for this type of software.

\section{$5 \quad$ Acknowledgment}

This paper is a project of the humanities and social science project of shandong university of higher learning: traditional culture of film and animation expression research (project number: J17RB037) periodic results.

\section{References}

[1] Calverley, G., Shephard, K. (2003). Assisting the uptake of on-line resources: why good learning resources are not enough, Computers \& Education, 41(3), 205-224. https://doi.org/10.1016/S0360-1315(03)00028-9

[2] Ray, R.D. (1995). Mediamatrix: an authoring system for adaptive hypermedia teaching learning resource libraries, Journal of Computing in Higher Education, 7(1), 44-68. https://doi.org/10.1007/BF02946142

[3] Leung, L. (1998). Lifestyles and the use of new media technology in urban china 1, Telecommunications Policy, 22(9), 781-790. https://doi.org/10.1016/S0308-5961(98)00055-X 
[4] Sabti, A.A., Chaichan, R.S. (2014). Saudi high school students' attitudes and barriers toward the use of computer technologies in learning English, Springer plus, 3(1), 1-8. https://doi.org/10.1186/2193-1801-3-460

[5] Banea, C., Mihalcea, R., Wiebe, J. (2013). Porting multilingual subjectivity resources across languages, IEEE Transactions on Affective Computing, 4(2), 211-225. https://doi.org/10.1109/T-AFFC.2013.1

[6] Wang, J., Spencer, K., Xing, M. (2009). Metacognitive beliefs and strategies in learning Chinese as a foreign language. System, 37(1), 46-56. https://doi.org/10.1016/j.system. $\underline{2008.05 .001}$

[7] Hansell, M. (2008). Teaching and learning Chinese as a foreign language: a pedagogical grammar by Xing, Janet Zhiqun, Modern Language Journal, 92(2), 331-332. https://doi.org/10.1111/j.1540-4781.2008.00729 9.x

[8] Li, S. (2007). Teaching and learning Chinese as a foreign language, Janet Zhiqun Xing, Studies in Second Language Acquisition, 29(4). https://doi.org/10.1017/S027226 3107070593

[9] Xu, Y., Chang, L.Y., Zhang, J., Perfetti, C. A. (2013). Reading, writing, and animation in character learning in Chinese as a foreign language, Foreign Language Annals, 46(3), 423444. https://doi.org/10.1111/flan.12040

[10] Xu, Y., Chang, L., Perfetti, C.A. (2014). The effect of radical-based grouping in character learning in chinese as a foreign language. Modern Language Journal, 98(3), 773-793. https://doi.org/10.1111/modl.12122

[11] Wong, L.H., Chai, C. S., Zhang, X., King, R.B. (2015). Employing the tpack framework for researcher-teacher co-design of a mobile-assisted seamless language learning environment, IEEE Transactions on Learning Technologies, 8(1), 31-42. https://doi.org/10.1109/ TLT.2014.2354038

[12] Mcandrew, P., Foubister, S.P., Mayes, T. (1996). Videoconferencing in a language learning application, Interacting with Computers, 8(2), 207-217. https://doi.org/10.1016/09535438(96)01028-4

[13] Kondo, M., Ishikawa, Y., Smith, C., Sakamoto, K., Shimomura, H., Wada, N. (2012). Mobile assisted language learning in university efl courses in japan: developing attitudes and skills for self-regulated learning, Recall, 24(2), 169-187. https://doi.org/10.1017/S095 8344012000055

[14] Tyler, A. (2010). Usage-based approaches to language and their applications to second language learning, Annual Review of Applied Linguistics, 30(30), 270-291. https://doi.org/10.1017/S0267190510000140

[15] Schwienhorst, K. (1998). The 'third place' - virtual reality applications for second language learning, Recall, 10(1), 118-126. https://doi.org/10.1017/S095834400000433X

\section{$7 \quad$ Author}

Xuezhou Liu (1969. 1), Shouguang, Master, Associate professor at Weifang University of Science and Technology, Weifang 262700, China. Research direction: Chinese traditional culture and film and television media. Published several core papers and published monographs. I have more than 20 years of experience in traditional culture teaching, and I have presided over six projects.

Article submitted 07 February 2018. Final acceptance 23 February 2018. Final version published as submitted by the author. 